\title{
SEARCH FOR OSCILLATIONS IN SOME AP STARS
}

J.A. Belmonte, P.L. Pallé, T. Roca Cortés

Instituto de Astrofísica de Canarias. Universidad de La

Laguna. Tenerife. Spain.

\begin{abstract}
Photometric observations of some Ap stars with a $50 \mathrm{~cm}$ telescope have been carried out at Izaña (Tenerife) in February 1986. The stars observed were, generally, magnetic - cool Ap stars. A search for global oscillations in the range 3 to 15 minutes has been undertaken and they seem to be present in some of the stars observed.
\end{abstract}

\section{OBSERVATIONS AND ANALYSIS.}

Photometric observations of some Ap stars have been carried out during February 1986. The telescope used was a $50 \mathrm{~cm}$, german mounting reflector sited at the Observatorio del Teide of the Instituto de Astrofisica de Canarias. In its Cassegrain focus there is the IAC.-UBV photometer used in these observations. All the observations have been made with a Johnson B filter of 4360 wavelength. Blocks of 58 data points taken each second with a time information were recorded each minute in magnetic tape for later analysis.

A list of the observed stars is presented in Table 1. Five of them are cool-magnetic Ap stars of the group IV (lines of $\mathrm{Sr}, \mathrm{Cr}, \mathrm{Eu}$ and other rare earths). In order to test our system a well known rapid oscillating Ap star, HD24712, was also observed. Figure 1a shows one of the light curves obtained in a dark night and good atmospheric conditions; in Figure $1 \mathrm{~b}$ the same is presented in terms of instrumental magnitudes as a function of time, starting at $0^{h}$ GMT of February the 6 th 1986.

Residual time series were obtained after reducing the data by standard least squares fitting to air mass $/ 1 /$. Figure 2 shows the power spectrum obtained via FFT to the residuals of the data of Figure 1. In the spectra of individual series, noise effects are important. Atmospheric transparency, (mostly eliminated by passing a low frecuency moving mean) and tracking effects become important. Average spectra for each star were obtained in order to avoid these problems. (Figure 3). Noise level is reduced and possible frecuencies related with global oscillations of the star can be discriminated.

Finally all individual daily series for a particular star were compacted in a single series. Iterative sine wave fitting was used to perform the spectral analysis. (Figure 4). 


\begin{tabular}{|c|c|c|c|c|c|c|c|c|}
\hline $\mathrm{HD}$ & (1950) & $(1950)$ & $\mathrm{m}_{\mathrm{v}}$ & $B-V$ & G & $\mathrm{N}$ & $t(\min )$ & $t_{M}(\min )$ \\
\hline $\begin{array}{r}22374 \\
24712 \\
40312 \\
47152 \\
81009 \\
98088 \\
112413\end{array}$ & 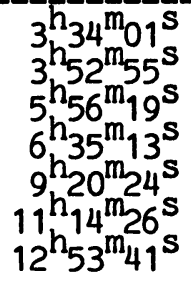 & $\begin{aligned} 23^{\circ} & 03^{\prime} \\
-12^{\circ} & 14^{\prime} \\
37^{\circ} & 12^{\prime} \\
29^{\circ} & 02^{\prime} \\
-09^{\circ} & 37^{\prime} \\
-06^{\circ} & 52^{\circ} \\
38^{\circ} & 35^{\prime}\end{aligned}$ & $\begin{array}{l}6.72 \\
6.00 \\
2.62 \\
5.79 \\
6.53 \\
6.14 \\
2.90\end{array}$ & $\begin{array}{r}0.13 \\
0.32 \\
-0.08 \\
0.00 \\
0.22 \\
0.21 \\
-0.12\end{array}$ & $\begin{array}{l}\text { IV } \\
\text { IV } \\
I \\
\text { IV } \\
\text { IV } \\
\text { IV } \\
\text { III }\end{array}$ & $\begin{array}{l}2 \\
7 \\
1 \\
4 \\
8 \\
5 \\
1\end{array}$ & $\begin{array}{l}180 \\
150 \\
207 \\
190 \\
265 \\
220 \\
180\end{array}$ & $\begin{array}{l}180 \\
229 \\
207 \\
305 \\
370 \\
305 \\
180\end{array}$ \\
\hline
\end{tabular}

TABLE 1. Journal of observations. HD catalogue number, 1950 medium equinox coordinates, magnitude and $\mathrm{B}-\mathrm{V}$ index. Ap standard Egret and Jascheck's classification group and the number of nights observed with the average interval time of observation and the length of the longest observing interval of each star are presented.
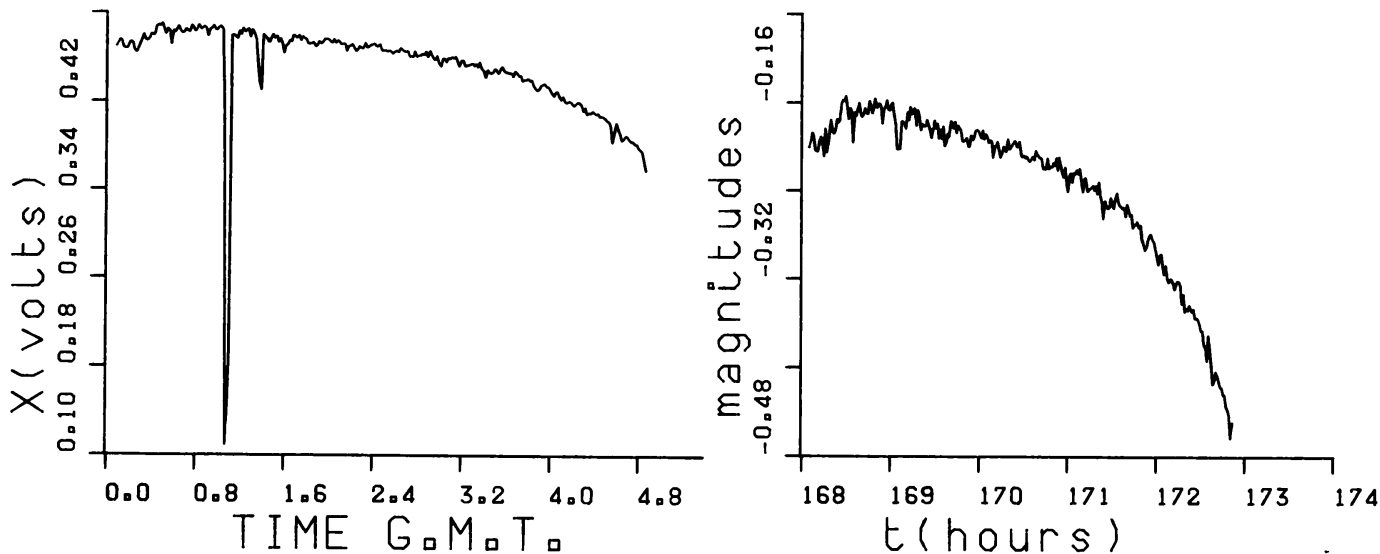

FIGURE 1a and 1b. Extinction curve of the star HD81009 for the night 12-13 February 1986. (1a). Id. as in figure 1a, after cleaning of anomalous points.(1b).

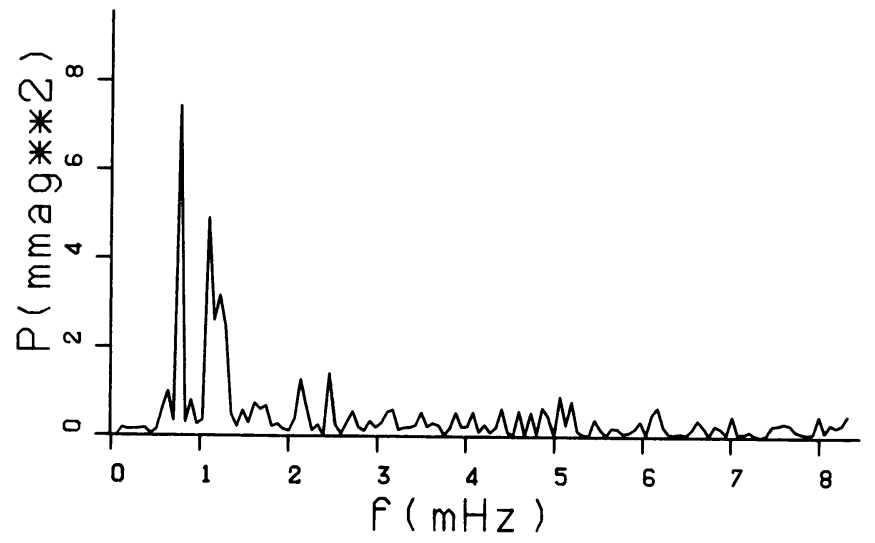

FIGURE 2. Power spectrum of the data shown in figure 1. It has been done using a FFT algorithm with a frecuency resolution of $65 \mathrm{microHz}$ and a Nyquist frecuency of $8.33 \mathrm{mHz}$. 


\section{RESULTS}

Table 2 gives the frecuencies of the modes that seem to be present in the 6 minutes peak of HD24712 in order to compare with those existing in literature $12 /$. Last results obtained from the spectral analysis of the first observing stars are finally presented in table 3.

\begin{tabular}{ccccc} 
& $\begin{array}{c}\mathbf{f} \\
\mathbf{m H z} \\
\mathbf{\pm . 0 0 2}\end{array}$ & $\begin{array}{c}\mathrm{P} \\
\text { min } \\
\mathbf{\pm . 0 1}\end{array}$ & $\begin{array}{c}\mathrm{A} \\
\mathbf{m m g} \\
\mathbf{\pm . 0 5}\end{array}$ & $\begin{array}{c}\mathrm{F} \\
\mathrm{rad} \\
\mathbf{\pm . 0 0}\end{array}$ \\
\hline $\mathrm{f}_{0}$ & 2.554 & 6.52 & 2.47 & 1.77 \\
$\mathrm{f}_{1}$ & 2.587 & 6.44 & 4.81 & -0.81 \\
$\mathrm{f}_{2}$ & 2.622 & 6.35 & 4.44 & -0.85 \\
$\mathrm{f}_{3}$ & 2.657 & 6.27 & 4.47 & -1.04 \\
$\mathrm{f}_{4}$ & 2.692 & 6.19 & 3.80 & -1.03 \\
$\mathrm{f}_{5}$ & 2.730 & 6.10 & 1.28 & -2.37 \\
$\mathrm{f}_{6}$ & 2.564 & 6.50 & 4.01 & -1.70 \\
\hline
\end{tabular}

TABLE 2. Modes of oscillation found in the 6 minutes range of the rapid oscillating Ap star HD24712.

\begin{tabular}{lllll} 
HD & \multicolumn{1}{c}{$f(\mathrm{mHz})$} & \multicolumn{1}{c}{$P(\mathrm{~min})$} & $\mathrm{A}(\mathrm{mmag})$ & $\mathrm{F}(\mathrm{rad})$ \\
\hline 22374 & $1.256 \pm .006$ & $13.26 \pm .03$ & $9.10 \pm .20$ & $1.17 \pm .08$ \\
& 1.961 & 8.50 & 5.10 & -1.37 \\
& 2.571 & 6.48 & 4.90 & 1.56 \\
47152 & 3.776 & 4.41 & 3.80 & 0.20 \\
81009 & $1.916 \pm .003$ & $8.70 \pm .02$ & $3.04 \pm .08$ & $0.78 \pm .05$ \\
& $1.565 \pm .002$ & $12.21 \pm .01$ & $2.50 \pm .05$ & $0.04 \pm .01$ \\
& 2.133 & 11.09 & 2.39 & -0.45 \\
& 2.599 & 7.81 & 2.03 & -0.19 \\
98088 & 2.947 & 6.41 & 2.17 & 0.69 \\
$9.102 \pm .002$ & 5.65 & 1.99 & 1.53 \\
& $3.03 \pm .01$ & $2.60 \pm .05$ & $0.72 \pm .05$
\end{tabular}

TABLE 3. Discovered peaks with a power well above the estimated noise level for each first observing cool-magnetic Ap star. The origin of time for the phase is $0^{\mathrm{h}}$ GMT of February $6^{\mathrm{th}} 1986$.

\section{ACKNOWLEDGEMENTS.}

The assistance of the maintenance service at Izaña is greatfully acknowledged.

\section{REFERENCES.}

/1/ Belmonte J.A., 1986 Tesis de Licenciatura. Universidad de Barcelona. /2/ Kurtz D.W., Seeman J., 1983 Mon. Not. R. astr. Soc., 205, 11. 


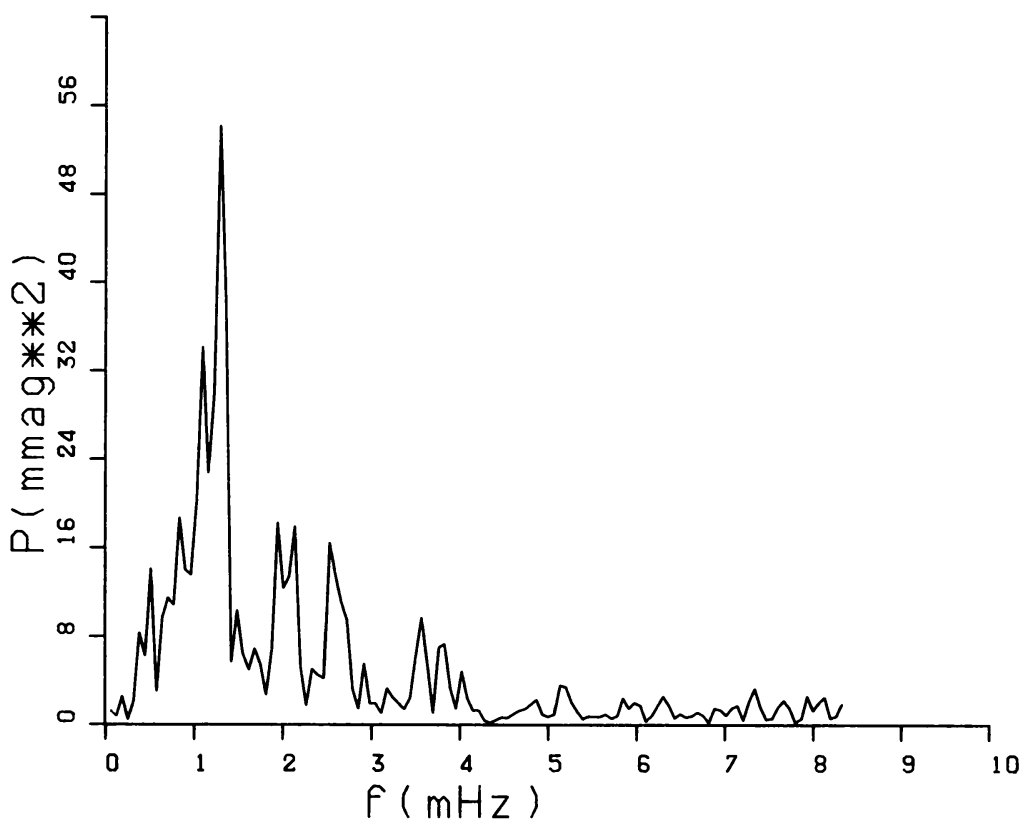

FIGURE 3. Average power spectrum of two good observing days of HD22374. Peaks with an amplitude well above the noise level $(1.9 \mathrm{mmag})$ seem to be present in the frecuency range of interest.

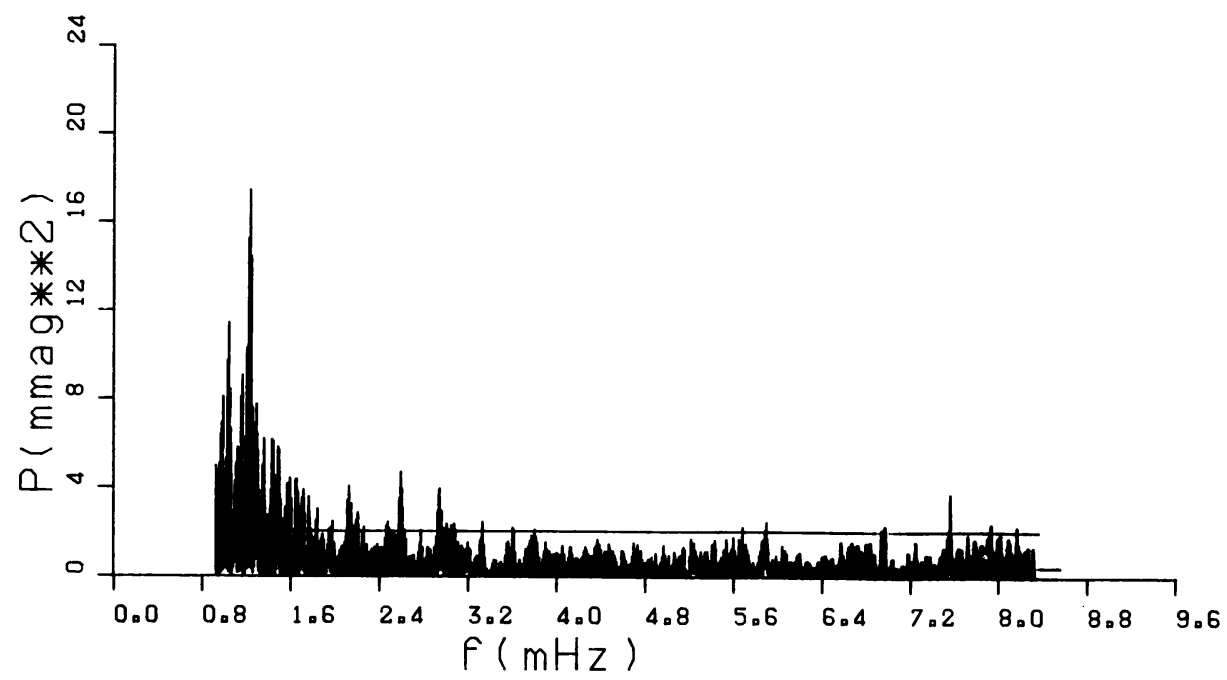

FIGURE 4. Power spectrum of the joint series of HD81009 corresponding to 32.18 hours of data from 8 nights of observation. Intrinsic resolution is $1.6 \mathrm{microHz}$. The straight line represents $3 \sigma$ noise level calculated from $1.4 \mathrm{mHz}$ to $8.33 \mathrm{mHz}$ and considered like a minimum cut-off on peaks amplitude associated with global oscillations of the stars. 